\title{
State-Dependent Dendritic Computation in Hippocampal CA1 Pyramidal Neurons
}

\author{
Sonia Gasparini and Jeffrey C. Magee \\ Neuroscience Center, Louisiana State University Health Science Center, New Orleans, Louisiana 70112
}

\begin{abstract}
Depending on the behavioral state, hippocampal CA1 pyramidal neurons receive very distinct patterns of synaptic input and likewise produce very different output patterns. We have used simultaneous dendritic and somatic recordings and multisite glutamate uncaging to investigate the relationship between synaptic input pattern, the form of dendritic integration, and action potential output in CA1 neurons. We found that when synaptic input arrives asynchronously or highly distributed in space, the dendritic arbor performs a linear integration that allows the action potential rate and timing to vary as a function of the quantity of the input. In contrast, when synaptic input arrives synchronously and spatially clustered, the dendritic compartment receiving the clustered input produces a highly nonlinear integration that leads to an action potential output that is extraordinarily precise and invariant. We also present evidence that both of these forms of information processing may be independently engaged during the two distinct behavioral states of the hippocampus such that individual CA1 pyramidal neurons could perform two different state-dependent computations: input strength encoding during theta states and feature detection during sharp waves.
\end{abstract}

Key words: CA1; dendrite; integration; nonlinear; sharp wave; theta

\section{Introduction}

The rodent hippocampus exhibits two major behaviorally related states, each displaying a characteristic neuronal population pattern. One of these states occurs as an animal actively explores the environment or during rapid eye movement sleep and is characterized by theta frequency $(5-10 \mathrm{~Hz})$ network oscillations (Vanderwolf, 1969; O'Keefe and Nadel, 1978). The other is a nontheta state that occurs during awake-immobility and slowwave sleep and is characterized by irregular large amplitude oscillations [sharp waves (SPWs)] and higher frequency (150-200 $\mathrm{Hz}$ ) synchronous discharges (ripples) (O'Keefe and Nadel, 1978; Buzsaki, 1986).

These separable behavioral states are also associated with distinct input-output patterns in the various hippocampal subregions, with the network appearing to operate at two distinctive timescales. Indeed, synaptic input to CA1 pyramidal neurons during theta is robust but spread out over hundreds of milliseconds, whereas, during SPWs, input synchrony is estimated to increase $\sim 10$-fold (Csicsvari et al., 1998, 2000). The output patterns produced during these two states are also quite different. During theta, the relative timing of action potential (AP) output advances over many tens of milliseconds as the animal moves toward and through the place field of a particular neuron

Received 0ct. 17, 2005; revised Dec. 29, 2005; accepted Dec. 30, 2005.

This work was supported by National Institute of Health Grant NS39458 (J.C.M.) and National Alliance for Research on Schizophrenia and Depression Grants YI2002 (S.G.) and II2003 (J.C.M.). We thank Gyorgy Buzsaki, Dan Johnston, and Randy Chitwood for many helpful discussions. We also express our gratitude to all of our friends and colleagues who offered us much needed kindness and support following the Hurricane Katrina disaster.

Correspondence should be addressed to Jeffrey C. Magee, Neuroscience Center, Louisiana State University Health Science Center, 2020 Gravier Street, New Orleans, LA 70112. E-mail: jmagee@|suhsc.edu.

DOI:10.1523/JNEUROSCI.4428-05.2006

Copyright $\odot 2006$ Society for Neuroscience $\quad$ 0270-6474/06/262088-13\$15.00/0
(O'Keefe and Dostrovsky, 1971; O'Keefe and Recce, 1993; Harris et al., 2002; Mehta et al., 2002). In contrast, an extremely precise and invariant form of output timing is found during SPWs, with AP firing found almost exclusively during a $1 \mathrm{~ms}$ time window of the ripple trough (Csicsvari et al., 1999).

We were interested in investigating the idea that a fundamental change in dendritic integration of synaptic input could provide a cellular-level mechanism supporting the state-dependent network activity. CA1 and other pyramidal neurons have been reported to be capable of performing either a linear form of integration (Margulis and Tang, 1998; Cash and Yuste, 1999; Nettleton and Spain, 2000; Tamas et al., 2002) or a nonlinear form stemming from the generation of dendritic spikes (Golding and Spruston, 1998; Larkum et al., 2001; Wei et al., 2001; Ariav et al., 2003; Gasparini et al., 2004; Polsky et al., 2004). Both of these integration styles appear to result from complex interactions among the passive and active dendritic membrane properties, as well as the properties of the synapses themselves. Some current hypotheses have compressed these two integration forms into a single sigmoid-shaped function with linear integration occurring simply because input levels are subthreshold for local spike generation (Poirazi et al., 2003; Polsky et al., 2004; Spruston and Kath, 2004). We propose here, however, that linear and nonlinear dendritic integration can be two fundamentally distinct integration forms, with each selectively engaged by input with different temporal structure. This situation could provide the network with a degree of flexibility that might be useful in producing the dramatically different timescales of network firing found during theta and SPWs. We have used multisite two-photon glutamate uncaging and simultaneous whole-cell recordings from the dendrites and soma of CA1 pyramidal neurons in acute hippocampal slices to determine whether different input patterns can produce 
distinct single-cell computations most complementary for a given behavioral state.

\section{Materials and Methods}

Transverse hippocampal slices ( $400 \mu \mathrm{m}$ thick) were prepared from 8- to 12-week old Sprague Dawley rats as described previously (Magee, 1998), according to methods approved by the Louisiana State University Health Science Center Institutional Animal Care and Use Committee. Rats were given a lethal dose of ketamine and xylazine, perfused through the ascending aorta with an oxygenated solution just before death, and decapitated.

Dendrites from pyramidal CA1 pyramidal cells were visualized using a Zeiss (Oberkochen, Germany) Axioskop fit with differential interference contrast (DIC) optics under infrared illumination. The external solution used for recordings contained the following (in mM): $125 \mathrm{NaCl}, 3 \mathrm{KCl}, 25$ $\mathrm{NaHCO}_{3}, 1.25 \mathrm{NaHPO}_{4}, 1.3 \mathrm{CaCl}_{2}, 1 \mathrm{MgCl}_{2}$, and 25 glucose, and was saturated with $95 \% \mathrm{O}_{2}$ and $5 \% \mathrm{CO}_{2}$ at $34-36^{\circ} \mathrm{C}$. Simultaneous wholecell patch-clamp recordings from distal dendrites and somata were performed using Dagan (Minneapolis, MN) BVC-700 amplifiers in the active "bridge" mode, filtered at $1-10 \mathrm{kHz}$ and digitized at $50 \mathrm{kHz}$. Patch pipettes had a resistance of 4-6 M $\Omega$ for dendritic and 2-4 M $\Omega$ for somatic recordings when filled with a solution containing the following (in mM): $120 \mathrm{~K}$-methylsulfate, $20 \mathrm{KCl}, 10$ HEPES, 0.5 EGTA, $4 \mathrm{NaCl}, 4$ MgATP, 0.3 Tris $_{2}$ GTP, 14 phosphocreatine, $\mathrm{pH}$ 7.25. The series resistance was usually $<20 \mathrm{M} \Omega$. The distance of the dendritic electrode from the visually identified soma was measured under DIC conditions.

EPSC-shaped waveforms characterized by AMPA current kinetics $\left(\tau_{\text {rise }}, 0.1 \mathrm{~ms} ; \tau_{\text {decay }}, 2 \mathrm{~ms}\right.$ ) (Magee and Cook, 2000; Smith et al., 2003) were injected through the whole-cell electrode. In particular, we injected either 10 identical EPSCs of increasing amplitude at different frequencies $(100 \mathrm{~Hz}$ to $10 \mathrm{kHz}$ ) or a random pattern of 12-260 EPSCs (of $80 \mathrm{pA}$ each) dispersed in 1-100 ms. The value of $80 \mathrm{pA}$ was chosen, because it generated a voltage deflection of $0.8-1 \mathrm{mV}$ when injected in the distal dendrites, as reported for the single synaptic event at these locations (Magee and Cook, 2000). In a set of experiments, a real-time conductance injection amplifier (SM-1; Cambridge Conductance, Cambridge, UK) (Harsch and Robinson, 2000) was used to stimulate the neurons by delivering a combination of AMPA and NMDA conductance changes (AMPA/ NMDA amplitude ratio was set at 2) (McDermott et al., 2005). In this case, two dendritic electrodes were used, to independently inject current and record the $V_{\mathrm{m}}$. The amplifier reproduced the voltage dependence of the block by $\mathrm{Mg}^{2+}$ by multiplying the linear conductance equation by a Boltzmann-type nonlinearity (Harsch and Robinson, 2000), where $K_{1}$ and $K_{2}$ were set to 0.3 and $0.06 / \mathrm{mV}$, respectively. $\tau_{\text {rise }}$ and $\tau_{\text {decay }}$ for NMDA currents were set to 1 and $20 \mathrm{~ms}$, respectively, and the reversal potential for AMPA and NMDA currents was set to $0 \mathrm{mV}$.

The presentation of variable spatiotemporal input patterns was performed using a dual galvanometer-based scanning system to multiphoton photorelease glutamate at multiple dendritic spines and simultaneously image local $\mathrm{Ca}^{2+}$ in the dendrites of CAl neurons (Prairie Technologies, Middleton, WI). Neurons were filled with Oregon Green BAPTA-1 (OGB-1) (Molecular Probes, Eugene, OR) (standard internal solution with EGTA replaced by $100 \mu \mathrm{M}$ OGB-1) and imaged using a $60 \times$ objective (Olympus, Melville, NY). Ultrafast, pulsed, laser light at $930 \mathrm{~nm}$ (Mira 900F; Coherent, Auburn, CA) was used to excite the OGB-1, while $720 \mathrm{~nm}$ ultrafast, pulsed, laser light (Chameleon; Coherent, Auburn, CA) was used to photolyze 4-methoxy-7-nitroindolinyl-caged L-glutamate (MNI-Glu) (Tocris Cookson, Ballwin, MO) (10 mm applied via pipette above slice) (Smith et al., 2003). To quantify input relative to unitary synaptic input for this region, MNI-Glu was uncaged at 7-10 identified spines ( $0.2 \mathrm{~ms}$ exposure) with a $100 \mathrm{~ms}$ uncaging interval at a given laser intensity. This exposure duration produced glutamate uncaginginduced EPSPs (glu-EPSPs) whose kinetics matched closely those reported for unitary excitatory input to this region. glu-EPSPs within 10 $\mu \mathrm{m}$ of the recording electrode had a $20-80 \%$ rise time of $0.5 \pm 0.04 \mathrm{~ms}$ and a decay time constant of $8.3 \pm 0.8 \mathrm{~ms}(n=22)$, comparable to a $\sim 0.6$ $\mathrm{ms}$ rise time and $7 \mathrm{~ms}$ decay time constant for distal-trunk miniature EPSPs (Magee and Cook, 2000). Locations were chosen to give a relatively uniform glu-EPSP amplitude $(2-3 \mathrm{mV}$ at the dendrite; $\sim 0.5 \mathrm{mV}$ at the soma) representing approximately three unitary synaptic inputs (each of $0.8 \mathrm{mV}$ at dendrite, $0.2 \mathrm{mV}$ for the soma) (Magee and Cook, 2000) for each location, giving a total of 20-30 for all 7-10 locations. Laser power was gradually increased to ultimately provide the equivalent of 60-120 synapses worth of input (where each individual glu-EPSP had an amplitude of 5-10 $\mathrm{mV}$ at the dendrite or $1-2 \mathrm{mV}$ at the soma) (see Fig. $2 b$ ). Two different temporal patterns $(0.3-0.4$ or 5.2 ms uncaging intervals) were then given at the respective laser power intensities, and this procedure was repeated for the different spatial distributions (all input spines within a dendritic region of 20 or $150 \mu \mathrm{m}$ ). Our uncaging scanner was measured to make a highly reproducible step across the entire imaging field $(\sim 130 \mu \mathrm{m})$ in $<300 \mu \mathrm{s}$. We generally used three to four steps to cover the full distance of the spatial profile with the remaining four to seven steps used to fill in the intervening space randomly (see Fig. $2 a$ ). The extraordinarily high spine density of the distal trunk region $(\sim 7 /$ $\mu \mathrm{m})$ made finding more than one or two adequately isolated spines impossible (Smith et al., 2003). This means that there was a significant glutamate spillover between some spines, with a larger than normal NMDA receptor-mediated slow EPSP component resulting. The functional impact of this was a larger amount of summation during the asynchronous input patterns (observable as an approximately twofold larger slope in Fig. $2 e$ than in Fig. 1e).

Data are reported as means \pm SEM. Statistical comparisons were performed by using Student's $t$ test. Means were considered to be significantly different when $p<0.05$.

\section{Results \\ Dendritic integration mode is dependent on the temporal input pattern}

To investigate the impact of temporal input pattern on dendritic integration, a random pattern of 12-260 synaptic inputs were delivered over varying durations of time (1-100 ms) through a dendritic electrode placed at $>250 \mu \mathrm{m}$ from the soma (Fig. 1a) (unitary conductance or current produced an $\sim 1 \mathrm{mV}$ dendritic EPSP that is similar in size and shape to the mean unitary EPSP found in this dendritic region) (see Materials and Methods) (Magee and Cook, 2000). Membrane potential from either nearby dendrite, soma, or both was simultaneously recorded with a second or third electrode.

When progressively increasing amounts of asynchronous input were delivered to the dendrite (input spread over time windows ranging from 10 to $100 \mathrm{~ms}$ ), the level of resulting depolarization increased in an approximately linear manner, with a slope that was dependent on the amount of input synchrony (Fig. 1a,e). Eventually, enough input was delivered to generate AP output. For all cases of asynchronous input patterns (10-100 ms), APs were first initiated in the soma/axon region of the neuron and then backpropagated into the dendrite (bAP) (Fig. 1c). Although the arrival of bAPs makes a precise quantification difficult, it appeared that dendritic depolarizations continue to increase as a linear function of input well beyond the output AP threshold (Fig. 1 $a, e, f$ ). Thus, as long as the input pattern was spread over tens of milliseconds, the dendrite integrated this input in a remarkably linear manner, producing progressively larger, slow depolarizations that directly reflected the number of inputs. The slow depolarizations propagated well to the soma where they initiated an action potential output at the soma/axon region. These data show that asynchronous input is associated with a linear integration mode that evokes action potential output from the soma/axon region.

A different pattern of synaptic input produced a different form of dendritic depolarization. When increasing amounts of highly synchronized input (all input arriving within 1-3 ms) were delivered to the dendrite, progressively larger, quite rapid, dendritic depolarizations were produced (Fig. 1b,e). Eventually, 


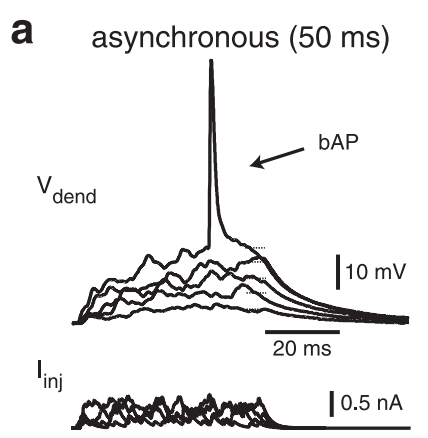

b synchronous (1 $\mathrm{ms})$
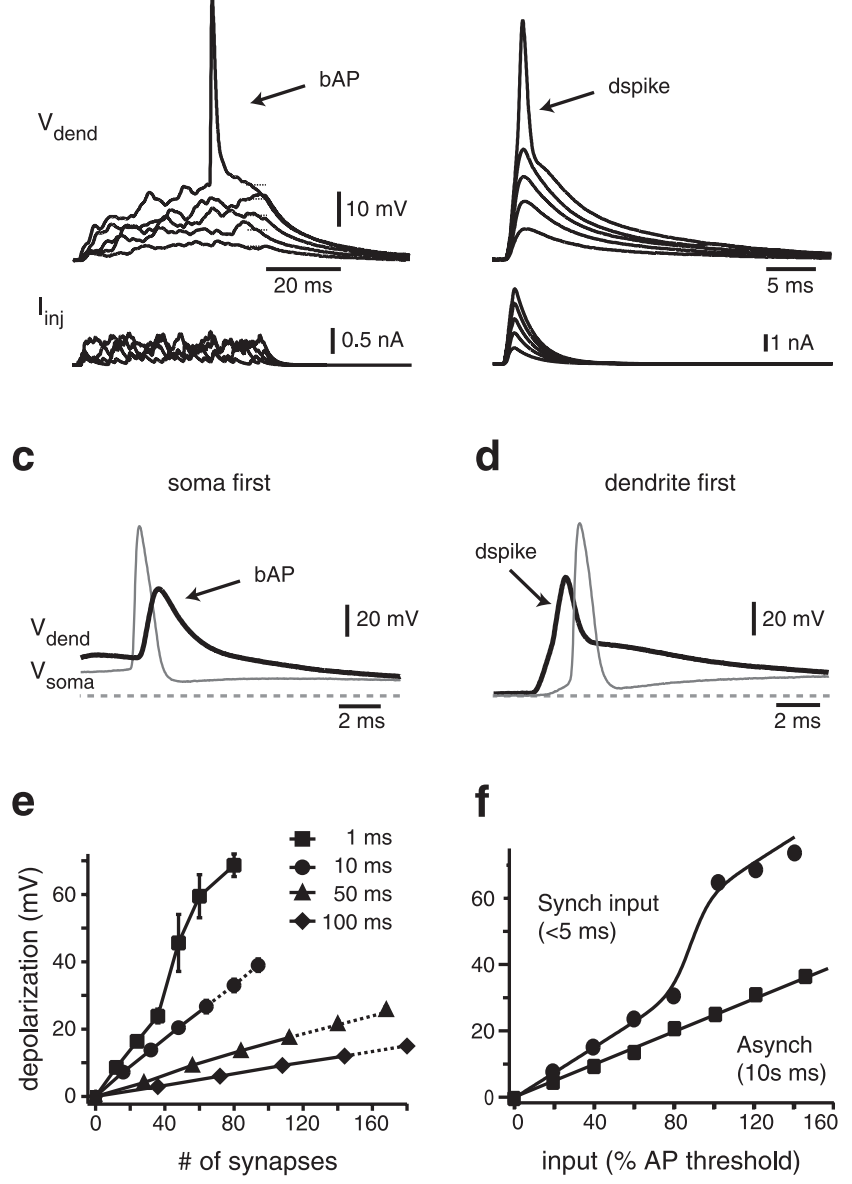

Figure 1. Linear and supralinear integration of synaptic inputs in CA1 pyramidal neurons. $\boldsymbol{a}$, Dendritic recordings showing the random pattern of input $\left(l_{\text {inj }}\right)$ and the resulting dendritic depolarization $\left(V_{\text {dend }}\right)$ produced by this input. At left ( $\boldsymbol{a}$; blue traces), the input pattern is asynchronous with the number of inputs increasing from 28 to 140 over a time window of $50 \mathrm{~ms}$. At right ( $\boldsymbol{b}$; black traces), the input pattern is a highly synchronous pattern in which the number of inputs, increasing from 12 to 60 , are delivered within $1 \mathrm{~ms}$. The dotted lines indicate the region in which the peak amplitude was calculated (average of the last $20 \%$ of the current injection). $c$, Expanded dendritic and somatic recordings from a neuron receiving suprathreshold asynchronous input (50 ms time window) showing that APs are always initiated in the soma/axon region, while synchronous input ( $\boldsymbol{d} ; 1 \mathrm{~ms}$ time window) causes dendritic spike generation that precedes somatic AP output. $\boldsymbol{e}$, Mean input- output relationships for the population of dendritic recordings $(n=9)$. The curves show that the shift from linear to supralinear summation occurred only for the most synchronous input. The data from suprathreshold 10-100 ms input are shown in dashed lines and are approximate because of bAPs. Error bars indicate SE. $\boldsymbol{f}$, Representative single neuron integration functions for synchronous (Synch) and asynchronous (Asynch) input patterns ( 1 and 50 ms input windows from the cell shown in $\boldsymbol{a}$ and $\boldsymbol{b}$ ). Input amount expressed as a percentage of the amount of input required to reach output AP threshold. Synchronous input data were fit by a sigmoid-like function in the form of $y=25 /(1+$ $\exp (-(n-88) / 5.1))+(0.38 n+0.4)$, whereas the asynchronous input data were fit by a linear function in the form of $y=0.24 n+0.1$.

however, enough synchronous input was delivered to evoke a local dendritic spike that clearly occurred before the initiation of an output spike at the soma/axon (Fig. 1d) (dendritic spike threshold was $52 \pm 5$ inputs for a temporal window of $<3 \mathrm{~ms}$ ). The occurrence of a dendritic spike produced a disproportionate increase in dendritic depolarization that resulted in a sigmoidshaped local input-output curve (Fig. 1e,f). We thus define this dendritic depolarization profile as a nonlinear form of integration.

Because the dendritic spike threshold in CA1 pyramidal neu- rons is heavily dependent on the rate of depolarization $(d V / d t)$, only tightly synchronized input can produce the rapid depolarization required for local dendritic spike initiation (Gasparini et al., 2004). Thus, the highly nonlinear form of integration produced by local spike generation is seen only in response to synchronous input patterns. Indeed, we never observed the initiation of dendritic spikes for the more asynchronous input patterns even when very large amounts of input were delivered to the dendrite (e.g., $>220$ inputs over $50 \mathrm{~ms}$ producing dendritic depolarizations to approximately $-25 \mathrm{mV}$ ). It should be noted that this level of depolarization is above threshold for both axonal APs and dendritic spikes evoked by synchronous input. Together, then, the data indicate that the form of dendritic integration (either linear or nonlinear) (Fig. $1 f$ ) is highly dependent on the timing of the input, with the dendrite performing an approximately linear integration for all input patterns except those that are extraordinarily well synchronized.

\section{Dendritic integration mode is dependent on the spatial input pattern}

To investigate how the spatial distribution of the input affects dendritic integration, we used multisite two-photon glutamate uncaging to deliver varying amounts of input in a spatial pattern that was either clustered or distributed. An input was considered to be clustered when a pattern of 7-10 uncaging events were delivered to spines covering $\sim 20 \mu \mathrm{m}$ of dendrite, whereas the distributed input was delivered to spines spread over 125-150 $\mu \mathrm{m}$ of dendrite. To mimic a progressive increase in the number of synaptic inputs for each of these spatial patterns, laser intensity was gradually enhanced (see Materials and Methods), and the resulting membrane potential deflections were recorded with a dendritic pipette located within or near the input. The impact of spatial distribution was determined for both synchronous (all input within $3 \mathrm{~ms}$ ) and asynchronous (all input within $50 \mathrm{~ms}$ ) input patterns.

For asynchronous input patterns, the effect of spatial distribution was minimal, with the only observable impact being a statistically insignificant decrease in the slope of the local input-output curve for the more dispersed inputs (clustered, $0.33 \pm 0.03$ $\mathrm{mV} /$ input, $n=5$; distributed, $0.28 \pm 0.03 \mathrm{mV} /$ input, $n=4$ ) (Fig. $2 c, e)$. For synchronous input patterns, the impact of spatial distribution was more dramatic. When all input was delivered to spines covering an $\sim 20 \mu \mathrm{m}$ region of the dendrite, a strongly nonlinear form of integration was observed as the highly synchronous and clustered input initiated a powerful local dendritic spike (amplitude, $64 \pm 3 \mathrm{mV}$; maximum spike $d V / d t$, $57 \pm 5 \mathrm{~V} / \mathrm{s}$; $n=8$ ) (Fig. $2 d, e$, blue traces). When the same temporally synchronous input pattern was delivered to spines in a distributed spatial pattern, the degree of nonlinear integration generated was either greatly reduced or removed completely (amplitude, $52 \pm 5$ $\mathrm{mV}$; maximum spike $d V / d t, 36 \pm 4 \mathrm{~V} / \mathrm{s} ; n=6$ ) (Fig. $2 d$,e, red traces). Thus, for synchronous input patterns, the mode of dendritic integration was highly dependent on the degree of spatial clustering of the input, with the dendrite producing a strong nonlinearity only if the input arrived within a spatial distribution of $<150 \mu \mathrm{m}$.

These data indicate that, depending on the spatiotemporal pattern of the synaptic input, either a linear or nonlinear form of dendritic depolarization occurs before the initiation of an output $\mathrm{AP}$ at the soma/axon region. We refer to these different dendritic depolarization forms as two distinct input pattern-dependent modes of integration, which are produced as the different spatiotemporal properties of the synaptic input differentially engage 
a

-distributed $\mathrm{w} / \mathrm{i} \sim 150 \mu \mathrm{m}$
-clustered $\mathrm{w} / \mathrm{i} \sim 25 \mu \mathrm{m}$

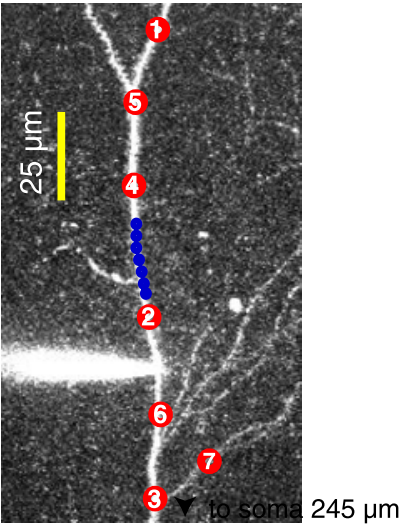

C

asynchronous

input (50 ms)

clustered

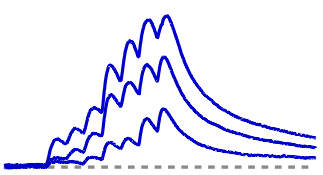

distributed

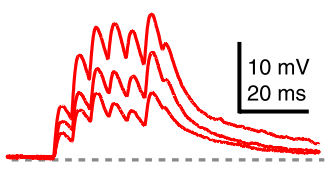

\section{b} clustered distributed

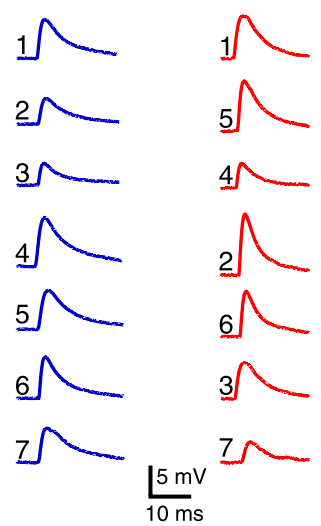

d synchronous input (3 ms)

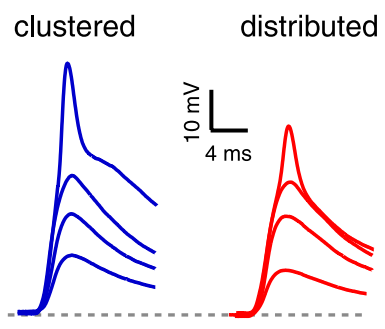

$\mathrm{dV} / \mathrm{dt}$

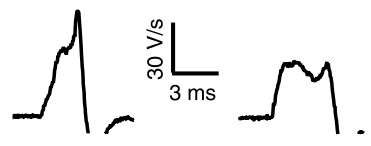

\section{e}

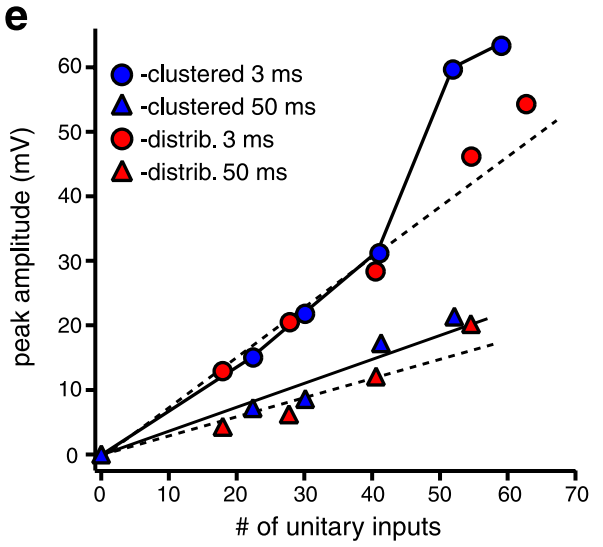

Figure 2. The spatial distribution of the input pattern determines dendritic integration. $\boldsymbol{a}$, Image stack of a distal apical dendrite region showing the experimental configuration for clustered (blue dots) and distributed (red dots) uncaging locations (recording pipette at $\sim 275$ $\mu \mathrm{m})$. w/i, Within. $\boldsymbol{b}$, Individual glu-EPSPs for the seven different sites with position indicated by associated number. c, Inputs given at positions $1-7$ with a $5.1 \mathrm{~ms}$ interval at three different laser intensities. $\boldsymbol{d}$, Inputs given at positions $1-7$ with a 0.4 ms interval at four different laser intensities. The bottom traces represent the temporal derivatives of dendritic spikes for clustered (left) and distributed inputs (right). $\boldsymbol{e}$, Input- output curves recorded at the dendrite for clustered and distributed (distrib.) input patterns. Note that only the synchronous pattern that is

the active properties of CA1 pyramidal dendrites. It seems likely that the differential shaping of the incoming synaptic input will in turn cause unique patterns of AP output to be associated with the linear and nonlinear integration modes.

\section{Input efficacy depends on the dendritic integration mode}

To investigate the impact of integration form on AP output, we first determined how the efficacy of the input, as indicated by the number of synaptic inputs required for axonal output, varied as a function of the different input patterns. Different input patterns were delivered as above (Fig. $3 b$ ), and we found that the number of synaptic inputs required for the generation of an axonal output was reduced for more synchronous when compared with less synchronous input patterns ( $53 \pm 3, n=18$, for $1-3$ ms duration vs $126 \pm 6$ for 50 ms duration, $n=18$ ) (Fig. $3 c-g$ ). Furthermore, we observed that distributing a highly synchronous input over $125-150 \mu \mathrm{m}$ of dendrite reduced the efficacy of the input toward that of the less synchronous input (clustered, $53 \pm 3$ inputs, vs distributed, $79 \pm 4$ inputs; $n=8$ and 6 , respectively) (Fig. 3f,g). These data show that CA1 pyramidal neurons preferentially respond to synchronous input patterns only if this input is spatially clustered enough to shift the dendrites into the nonlinear integration mode. Presumably, this effect is attributable to the initiation of a dendritic spike by this pattern of input.

We tested the idea that the generation of a strong dendritic spike is required for increasing the efficacy of a synchronous and clustered input by reducing dendritic excitability through bath application of a low concentration of tetrodotoxin (TTX) (50 nM). Such low concentrations of TTX have repeatedly been shown to effectively dampen dendritic excitability without greatly affecting that of the somatic/axonal region (somatic action potential threshold for $1 \mathrm{~ms}$ input: control, $-54 \pm 1 \mathrm{mV}$; TTX, $-49 \pm 1 \mathrm{mV}$; for $50 \mathrm{~ms}$ input, control, $-52 \pm 1 \mathrm{mV}$; TTX, $-47 \pm 1 \mathrm{mV}$ ) (Magee and Carruth, 1999; Metz et al., 2005). TTX application completely eliminated the ability of the dendrite to produce the supralinear integration mode that is usually associated with synchronous input without changing the dendritic depolarization profile produced by less synchronous input (data not shown).

In this low TTX condition, the number of inputs required for an axonal output dramatically increased for the most synchronous input pattern (132 \pm 10 inputs within $1 \mathrm{~ms} ; n=9)$ but increased only slightly for the less synchronous patterns (162 \pm 16 for the 50 ms duration; $n=12$ ) (Fig. $3 g$ ). A similar TTXdependent decrease in the efficacy of synchronous synaptic input has been observed in vivo (Canals et al., 2005). These data indicate that it is the robust propagation of the dendritic spike, not simply the synchronous input pattern itself, that is required for the generation of an axonal spike at lower input levels. In fact, the initiation and robust propagation of the dendritic spike make highly synchronous input the preferred pattern, despite the severe lowpass filtering properties of dendrites. Furthermore, the strict spatial requirements for dendritic spike generation allow CA1 pyramidal neurons to select for those patterns that are both synchronous and clustered. All other patterns will be less effective

$\leftarrow$

also clustered in space produces a strong nonlinearity. Distributing the synchronous input over $\sim 150 \mu \mathrm{m}$ reduced or removed the ability of the dendrite to shift into the nonlinear integration mode. Laser intensities produced the equivalent of 51 clustered and 53 distributed synapses in $\boldsymbol{b} ; 30,41$, and 51 clustered synapses and 28, 40, and 53 distributed synapses in $\boldsymbol{c}$; and 22,30,41, and 51 clustered synapses and 18,28,40, and 53 distributed synapses in $\boldsymbol{d}$. 


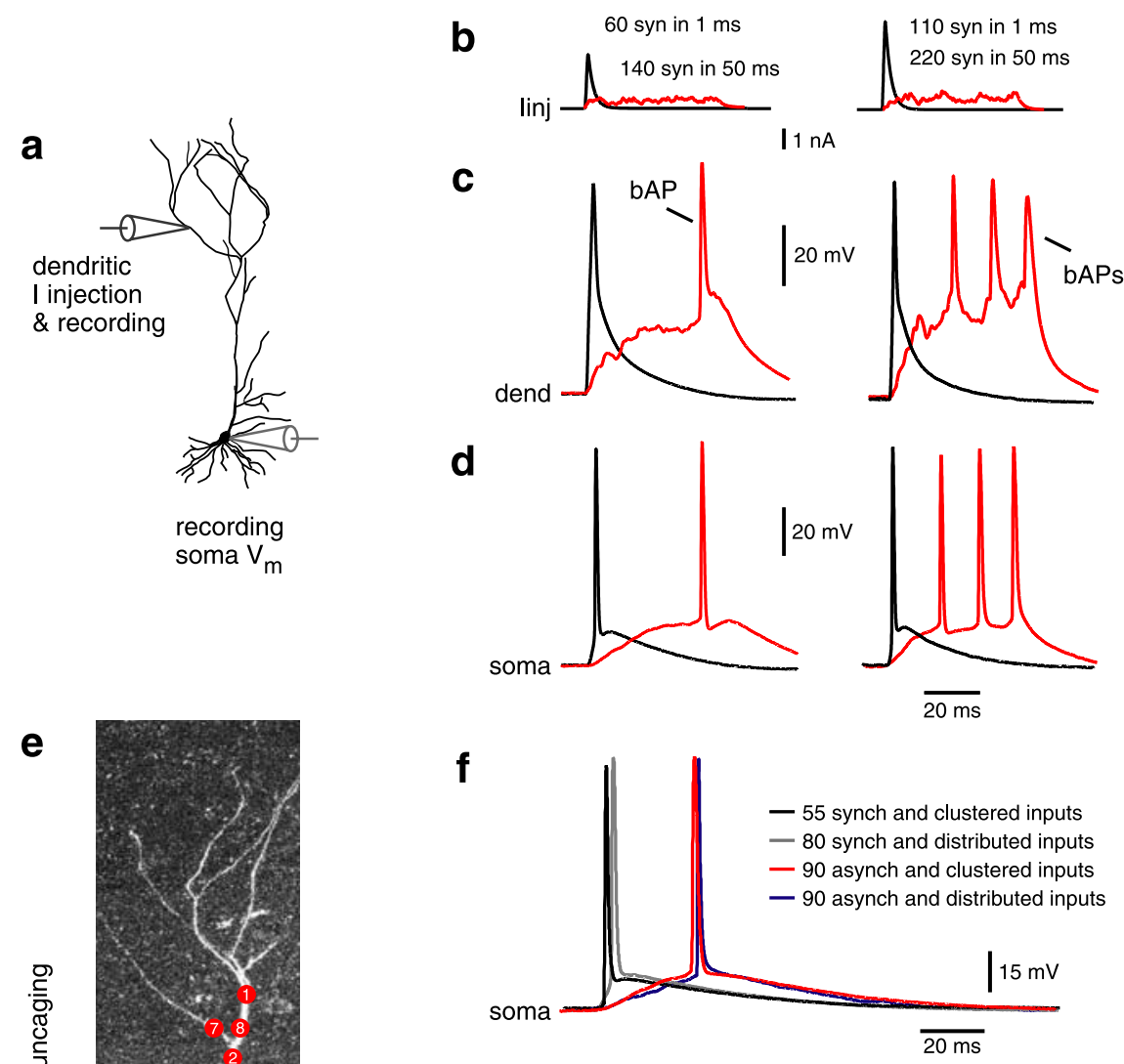

g

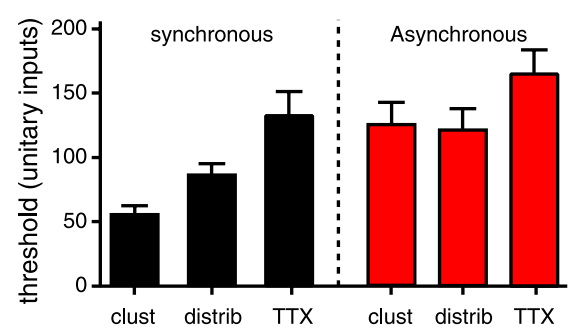

h

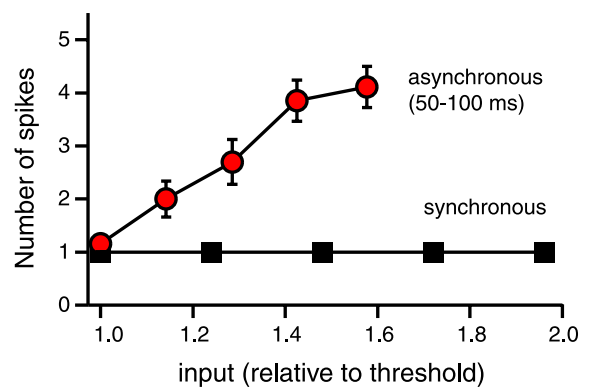

Figure 3. Dendritic integration mode determines input efficacy and spike rate variability. $\boldsymbol{a}$, Experimental configuration. A dendritic whole-cell electrode was used to inject a random pattern of EPSC-shaped currents while the corresponding voltage deflections were recorded with both dendritic and somatic electrodes. $\boldsymbol{b}$, Examples of the random patterns of EPSC-shaped currents that were injected into the dendrite in the case of synchronous ( $\leq 1 \mathrm{~ms}$; black) and asynchronous ( $50 \mathrm{~ms}$; red) input patterns. $\boldsymbol{c}, \boldsymbol{d}$, Dendritic (dend; $\boldsymbol{c}$ ) and somatic (soma; $\boldsymbol{d}$ ) recordings showing that higher input levels are required for output generation in the case of dispersed synaptic activation. Traces to the right in $\boldsymbol{b}$ - $\boldsymbol{d}$ demonstrate the effect of increasing the amount of input to nearly twice threshold levels. $\boldsymbol{e}$, Image stack of a CA1 pyramidal neuron showing the experimental configuration. Red dots indicate the approximate locations of the spines that were used for the distributed input pattern. Clustered pattern was given to spines located within an $\sim 20 \mu \mathrm{m}$ region near the scale bar. $f$, Somatic membrane potential recordings showing that the number of inputs required to achieve action potential output differs depending on the spatial and temporal distribution of the input pattern. The patterns that were both synchronous and clustered required the fewest inputs. synch, Synchronous; asynch, in producing an action potential output, because they require significantly greater amounts of input.

We next examined the impact that increasing the amount of input above threshold has on AP output. As expected, the number of APs evoked by asynchronous input patterns increased as the level of input was enhanced beyond threshold (Fig. $3 b-d, h$ ). Conversely, the initiation of a dendritic spike by the synchronous input pattern almost always resulted in only a single AP generated at the soma even at input levels that were twice threshold. These data indicate that the maintenance of the linear integration mode above AP output threshold allows the number of action potentials initiated by CA1 pyramidal neurons to steadily change as a function of input levels during asynchronous or spatially distributed input patterns. In contrast, the transition into the nonlinear integration mode greatly reduced the variation in spike rate such that only a single action potential output was evoked in response to the highly synchronous and clustered input.

\section{Output precision is dependent on dendritic integration mode}

We found another aspect of action potential output that was dependent on the mode of dendritic integration. Not surprisingly, the latency of somatic action potential output decreases with increased input synchrony (from $41.5 \pm 1.3$ for the 50 $\mathrm{ms}$ interval to $2.8 \pm 0.1 \mathrm{~ms}$ for the $1 \mathrm{~ms}$ duration; $n=12$ ) (Fig. $4 a-d$ ). Furthermore, the less synchronous input produced an action potential output whose latency was quite variable from trial to trial, especially compared with that produced by the most synchronous and clustered input pattern. Indeed, the jitter (defined as the SD of spike latency) associated with the synchronous patterns was more than an order of magnitude less than that

$\leftarrow$

asynchronous. $\boldsymbol{g}$, Plot of the number of synapses required for output generation for synchronous input (all within $3 \mathrm{~ms}$ ) or asynchronous input that was either clustered (clust), distributed (distrib), or clustered plus $50 \mathrm{~nm}$ TTX. The plot shows that the neuron will preferentially respond to clustered and synchronous input, only. Clustered input includes input from current injections, conductance changes, and glutamate uncaging. Distributed was only from glutamate uncaging. TTX condition was for current injections and conductance changes. $\boldsymbol{h}$, Plot of number of output APs evoked expressed as a function of the amount of input (relative to threshold) for either asynchronous or synchronous input, showing that the number of output APs can significantly vary as a function of input number only for asynchronous input patterns. Error bars indicate SE. 
a
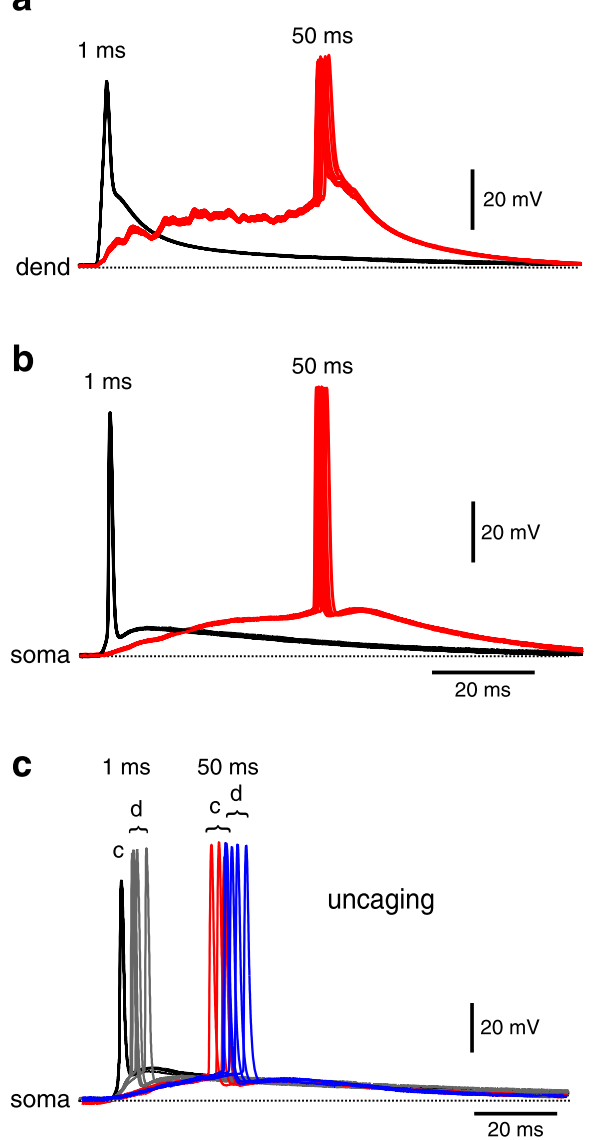

d

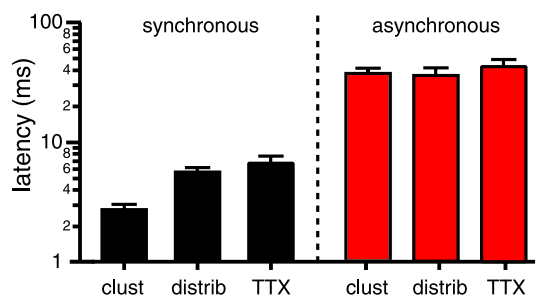

e

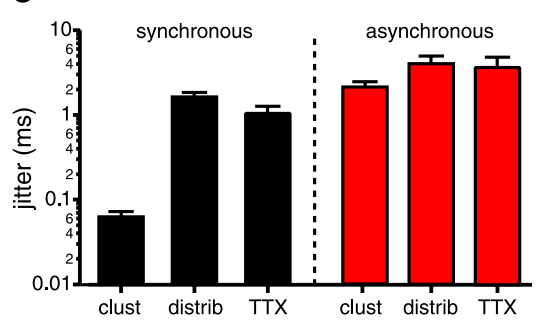

f

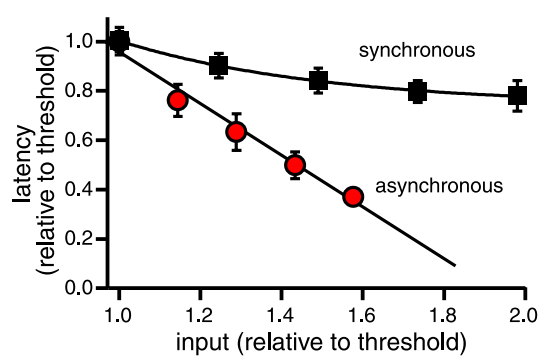

Figure 4. The dendritic integration mode determines the output timing and variability. $\boldsymbol{a}, \boldsymbol{b}$, Dendritic (dend; $\boldsymbol{a}$ ) and somatic (b) recordings showing 10 traces obtained for the same just suprathreshold random-patterned dendritic current injections for either synchronous (within $1 \mathrm{~ms}$ ) or asynchronous (within $50 \mathrm{~ms}$ ) input. c, Somatic recordings of just suprathreshold stimulation given by two-photon MNI-glu uncaging to spines in either a clustered ( $\sim 20 \mu \mathrm{m}$; labeled " $c$ ") or distributed ( $\sim 150 \mu \mathrm{m}$; labeled " $\mathrm{d}^{\prime \prime}$ ) spatial pattern for synchronous (within $3 \mathrm{~ms}$; black and gray traces) or asynchronous (within $50 \mathrm{~ms}$; red and blue traces) temporal patterns. $\boldsymbol{d}$, Plot of the latency to the first spike for either synchronous or asynchronous input delivered as clustered (clust) or distributed (distrib) spatial patterns. Also, the impact of $50 \mathrm{~nm}$ TTX application is shown. $\boldsymbol{e}$, Plot of action potential jitter (defined as the SD of the axonal action potential timing in repeated identical trials) for either synchronous or asynchronous input delivered as clustered or distributed spatial patterns. Also, the impact of $50 \mathrm{~nm}$ TTX application is shown. The plots show that the initiation of a dendritic spike for synchronous and clustered input disproportionately decreases latency and the variability of the action potential output timing. $\boldsymbol{f}$, Plot of AP latency (expressed as relative to the latency at just threshold values; see $\boldsymbol{e}$ ) versus the amount of input (relative to the threshold level) for both synchronous input (within $3 \mathrm{~ms}$ ) and asynchronous input (50 ms). Action potential latency was highly dependent on the level of input only when the input pattern was asynchronous, whereas latency was extremely invariant when synchronous input patterns were delivered. Error bars indicate SE.

of the asynchronous or distributed input patterns $(2.1 \pm 0.4 \mathrm{~ms}$, $n=11$, for the $50 \mathrm{~ms}$ duration, vs $0.06 \pm 0.01 \mathrm{~ms}, n=12$, for the $1 \mathrm{~ms}$ duration) (Fig. 4e). A similarly low jitter has been reported for APs initiated by basal dendritic spikes in CA1 pyramidal neurons once these more weakly propagating spikes are paired with subthreshold depolarizations (Ariav et al., 2003).

As above, inhibiting the ability of the dendrites to produce a nonlinear integration by spatially distributing synchronous input or by bath-applying low TTX greatly affected AP latency and precision (Fig. 4c). TTX (50 nM) more than doubled the latency of action potential output induced by synchronous input $(6.1 \pm 0.3$ $\mathrm{ms} ; n=6$ ) (Fig. 4e) while only slightly increasing AP latency for the more asynchronous patterns $(47.6 \pm 0.9 \mathrm{~ms} ; n=13)$. Likewise, the extremely low latency variation observed during highly synchronized input patterns was completely eliminated in the presence of $50 \mathrm{~nm}$ TTX (the jitter for the $1 \mathrm{~ms}$ duration increased to $0.9 \pm 0.1 \mathrm{~ms} ; n=6$ ) (Fig. $4 e$ ). The increased precision of action potential output was also abolished with input patterns that were spread over 125$150 \mu \mathrm{m}$ (jitter increased from $0.06 \pm 0.01$ to $1.4 \pm 0.1 \mathrm{~ms} ; n=6$ and 5 , respectively) (Fig. 4c,e). Again, this indicates that it is not simply the synchronized input pattern itself, but the shift into the nonlinear dendritic integration mode that is required for the extremely precise action potential output pattern associated with this input pattern. Indeed, it seems likely that it is the extraordinarily rapid depolarization of the soma/axon region (10's mV/ms) (Gasparini et al., 2004) by the actively propagating dendritic spike that allows the remarkably precise spike output. Thus, without the strong nonlinear dendritic integration mode, the neuron loses the uniquely precise action potential output normally associated with the arrival of highly synchronous and well clustered input patterns.

Finally, we again observed that only during asynchronous input patterns was the latency to first action potential firing capable of varying significantly as a function of the number of inputs (Fig. $4 f$ ). Indeed, the ability of the neuron to change spike timing as a function of input amount was severely reduced if the nonlinear integration mode was engaged by highly synchronous and clustered input. Thus, spike timing in CA1 pyramidal neurons can significantly vary as a function of the amount of input only when the dendrites integrate incoming input in a linear manner. If, in contrast, the input is highly clustered and synchronous such that a dendritic spike is initiated, AP output timing will be remarkably insensitive to variations in input strength.

Thus far, we have seen that, depending on the spatiotemporal input pattern, the dendrites of CA1 pyramidal neurons can perform two different forms of integration and that a unique action potential output pattern is associated with each of these integration modes. The linear integration mode was most frequently engaged (by a wide variety of less synchronous or spatially distributed input patterns), and it was associated with an output pattern in which the number and timing of evoked action potentials can vary as a function of input intensity. The other nonlinear dendritic integration mode was produced by synaptic input patterns that are both highly synchronized and spatially clustered and was associated with an output pattern characterized by a reduced output threshold and short latency, low jitter action potentials that were relatively unresponsive to changes in the number of inputs. We next wanted to examine the functional impact of these two distinct dendritic integration modes.

Dendritic integration mode during different behavioral states The hippocampus exhibits two distinct behavioral states, and as detailed below, each of these states is associated with a unique input-output pattern in area CA1. We were interested in deter- 
mining the role that the input-dependent modification of dendritic integration plays in producing the appropriate network patterns observed during each of these unique behavioral states. To this end, we presented CA1 pyramidal neurons with input patterns similar to those reported to be found during either theta or SPW states, determined whether the appropriate output pattern was produced, and examined the role of the dendritic integration mode in producing this output.

\section{Theta states}

During theta states, the synaptic input to CA1 pyramidal neurons increases with the movement of the animal toward and through the place field of that neuron (Harris et al., 2002; Mehta et al., 2002). In response, these neurons increase their firing rates and shift the timing of the spikes progressively forward over many tens of milliseconds (phase precession) (O'Keefe and Dostrovsky, 1971; O'Keefe and Recce, 1993). The magnitude and temporal pattern of synaptic input during this state can be estimated from the firing of the presynaptic CA3 pyramidal neuron population. The number of CA3 pyramidal cells that are firing during the $100-200 \mathrm{~ms}$ of a theta cycle has been estimated to be $3-5 \%$ of the total population (Csicsvari et al., 1998, 2000). Given the anatomical organization of the Schaffer collateral pathway, this works out to be $\sim 600$ potential synaptic inputs (4\% of 15,000 apical Schaffer collateral synapses) (Bannister and Larkman, 1995; Megias et al., 2001). Other factors, such as the total number of action potentials fired per cycle (from one to four per cycle) (Csicsvari et al., 2000) and the low probability of release at each synapse (0.1-0.5) (Raastad et al., 1992; Allen and Stevens, 1994; Smith et al., 2003), make it likely that a group of presynaptic CA3 pyramidal neurons provides a given CA1 pyramidal neuron with an input that ranges from $<100$ to $\sim 500$ inputs per cycle as the animal moves toward and through the place field of that cell. Simultaneous with this pattern of excitation, the proximal portions of CA1 pyramidal neurons (the soma and proximal trunk) receive a rhythmic 5-10 Hz input from the inhibitory basket cell and axo-axonic cell network (Fox, 1989; Cobb et al., 1995, Klausberger et al., 2003), whereas the dendrites receive a relatively sparse $(\sim 3 \%$ of excitatory synapse numbers) but nevertheless potentially effective input from the bistratified and O-LM (oriens-lacunosum moleculare) interneuron populations that is $\sim 180^{\circ}$ out of phase with the axo-axonic and basket cell input (Klausberger et al., 2004).

We therefore examined the pattern of action potential output resulting from the combination of a progressively increasing asynchronous excitatory input to the dendrite with an out-ofphase $10 \mathrm{~Hz}$ somatic hyperpolarizing sine wave to mimic proximal inhibitory input (amplitude was adjusted to increase approximately twofold the threshold amount of excitatory input) (Fig. $5 a, b$ ) (Harris et al., 2002; Mehta et al., 2002). The phasic dendritic input pattern was set up to mimic the progressive increase in excitation from 80 to 400 synaptic inputs, phasically interrupted by a like-sized dendritic inhibitory input (Klausberger et al., 2003, 2004). Generally, by the third cycle, the dendritic depolarization started to overcome the somatic inhibition and action potential output was generated (for 240 synapses activated in the example in Fig. 5a). As the input levels increased, the level of dendritic depolarization also increased accordingly as did the number of output APs per cycle (Fig. $5 a, d, e$ ). Together with this progressive increase in spike firing, the mean spike timing smoothly moved forward with respect to the theta phase demonstrating the characteristic phase precession found during theta states (Fig. $5 a, d, e$ ). Thus, the injection of asynchronous input
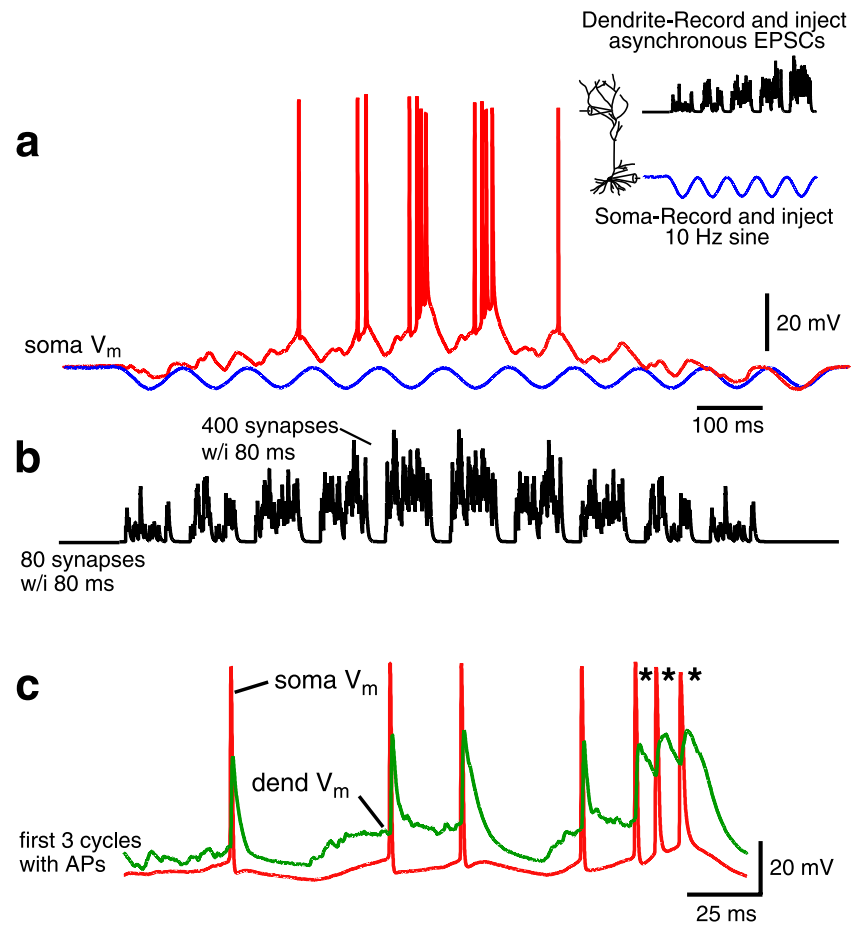

d

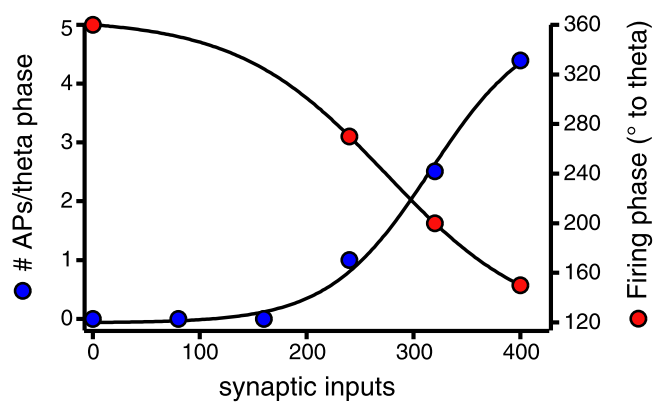

e

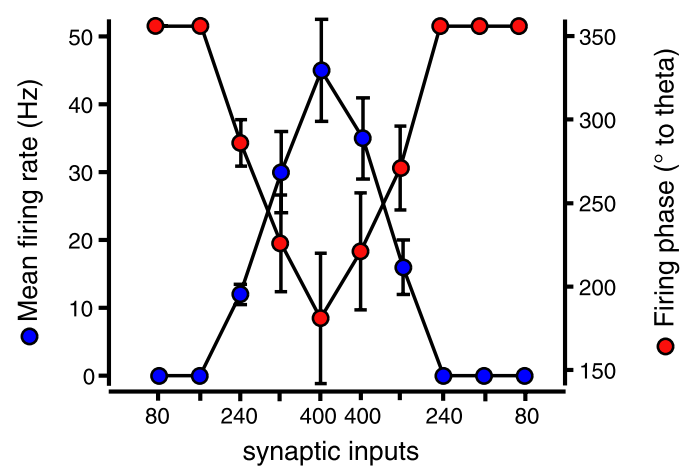

Figure 5. Linear dendritic integration during theta-like activity. $\boldsymbol{a}$, Somatic recording (red) obtained as a $10 \mathrm{~Hz}$ sine wave was injected in the soma (blue), and a random pattern of EPSC-shaped currents of increasing intensity (from 80 to 400 synapses in increments of 80 ) was simultaneously injected through a dendritic electrode $(\boldsymbol{b})$. c, Simultaneous somatic and dendritic recordings of the first three cycles with APs shown at an expanded timescale, demonstrating that the dendrite remained in the linear integration mode with bAPs only. The asterisks indicate the presence of a prolonged bAP duration presumably because of enhanced dendritic $\mathrm{Ca}^{2+}$ channel activation. $\boldsymbol{d}$, Plot of AP numbers and mean firing phase versus the amount of input delivered to the neuron shown in $\boldsymbol{a}$, demonstrating that the number of spikes generated at the soma increased with the number of synapses activated. In addition, the coarse timing of the spiking was advanced in relation to the $10 \mathrm{~Hz}$ sine wave. $\boldsymbol{e}$, Average plot of the mean firing rate and the firing phase as a function of the number of inputs $(n=6)$. Error bars indicate SE. 
a 1 synchronous dendritic input 60-100 synapses w/i $1 \mathrm{~ms}$

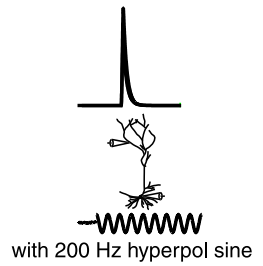

2

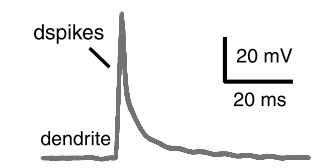

with $200 \mathrm{~Hz}$ hyperpol sine

3

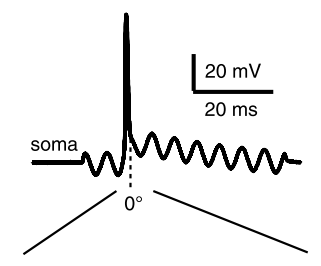

4

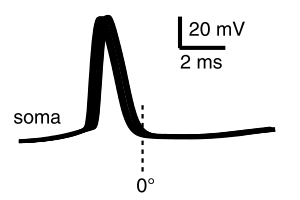

5

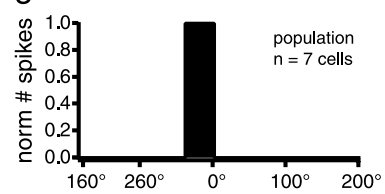

b asynchronous dendritic input 180 synapses $w / i 50 \mathrm{~ms}$
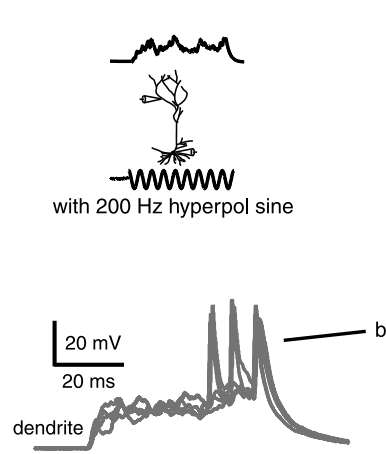

with $200 \mathrm{~Hz}$ hyperpol sine
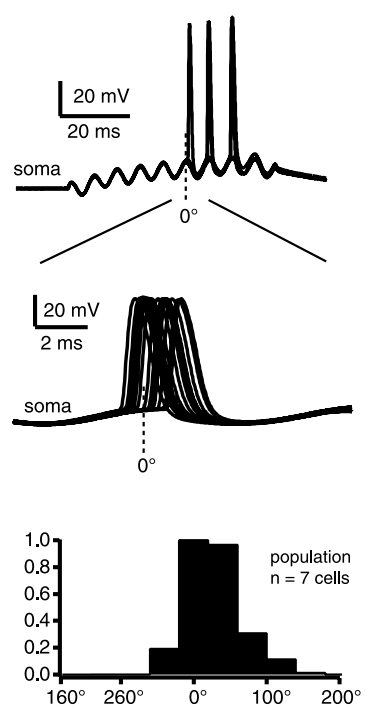

C asynchronous dendritic input 50-90 synapses w/i 4 ms

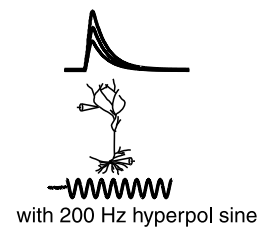

of the trials within those neurons), bAP duration increased significantly during the periods of highest input because of what is presumably an enhanced activation of dendritic voltage-gated $\mathrm{Ca}^{2+}$ channels (Fig. $5 c$, asterisks). These " $\mathrm{Ca}^{2+}$ shoulders" appeared to propagate to the soma where they produced an afterdepolarizing potential and burst firing (Golding et al., 1999; Magee and Carruth, 1999). Such an event is reminiscent of BAC (backpropagation-activated calcium spike) firing in layer $\mathrm{V}$ pyramidal cells (Larkum et al., 2001), except that here it requires more than a single spike (more than three bAPs) and is only likely to occur as the dendritic A-type $\mathrm{K}^{+}$current is inactivated by prolonged dendritic depolarizations (Hoffman et al., 1997; Magee and Johnston, 1997). The role of this firing pattern in theta activity will be further explored in future experiments.

\section{Sharp-wave states}

CA1 input-output features are quite different during sharp wave/ripple activity. Here, the level of input synchrony is elevated almost tenfold, with $\sim 4 \%$ of the CA3 pyramidal cell population providing input to CA1 within a $20 \mathrm{~ms}$ time window (Csicsvari et al., 2000). This synchronous input pattern is also associated with an action potential output reported to occur almost exclusively within a $1 \mathrm{~ms}$ time window during the troughs of the extracellularly recorded ripple event $(\sim 200 \mathrm{~Hz})$ of SPW states (jitter, $\sim 0.3 \mathrm{~ms}$ ) (Csicsvari et al., 1999). In an attempt to reproduce this in vivo situation, we paired a dendritic excitatory input of 60-100 synaptic inputs arriving within $1-3 \mathrm{~ms}(1.5 \%$ of 15,000 synapses with a mean $P_{\text {release }} \sim 0.3$ ) with a $200 \mathrm{~Hz}$ hyperpolarizing sine wave at the soma. Again the somatic sine wave is used as an approximation of the phasic arrival of proximal inhibition recorded during the ripple events (Fig. 6a1) (Buzsaki, 1986; Ylinen et al., 1995; Klausberger et al., 2003, 2004). This synchronous input pattern evoked a dendritic spike producing a well timed output (all output occurred within a patterns similar to those found during theta states left the dendritic arbor in a linear integration mode (only bAPs were observed) (Fig. $5 c$ ) and produced an output whose rate and coarse timing smoothly varied as a function of input intensity, just as occurs in the behaving animal (O'Keefe and Dostrovsky, 1971; O'Keefe and Recce, 1993; Harris et al., 2002; Mehta et al., 2002). These data indicate that, when paired with a phasic proximal inhibitory input, the linear dendritic integration mode is uniquely capable of producing an action potential output whose rate and coarse timing can directly reflect the amount of synaptic input received by the neuron.

Interestingly, in a number of cases ( $67 \%$ of neurons and $38 \%$

$1 \mathrm{~ms}$ time window) that was characterized by a short latency and high precision (jitter, $0.04 \pm 0.01 \mathrm{~ms} ; n=7$ ) (Fig. 6a2-a5). Importantly, this high level of precision was relatively insensitive to modifications that might be expected to occur quite commonly during in vivo conditions. That is, the variability in the timing of the output APs with respect to the $200 \mathrm{~Hz}$ sine was still very low even with changes in resting membrane $( \pm 5 \mathrm{mV})$ and the number of inputs (increases to twofold above threshold) (jitter, $0.16 \pm 0.04 \mathrm{~ms} ; n=5$ ) (Fig. 6a). The jitter in AP timing recorded under these conditions approaches that reported for in vivo recordings during SPWs (0.2 vs $\sim 0.3 \mathrm{~ms}$ ) (Csicsvari et al., 1999).

The recruitment of the nonlinear integrative properties of the 
dendrite by a synchronous input was absolutely required to produce the extremely well timed and invariant AP output during the simulated SPW states (Fig. 6a). When an asynchronous input ( 180 synapses activated within $50 \mathrm{~ms}$ ) was injected into the dendrites along with the $200 \mathrm{~Hz}$ hyperpolarizing somatic input, the threshold for spike generation was reached at the soma (Fig. 6b). As a consequence of the lower precision associated with the linear integration mode, the output spiking was spread over three cycles instead of a single (Fig. 6b3). Even when the spike timing was plotted relative to the phase of an individual cycle (to remove the cycle variations), the degree of temporal dispersion was much greater than that achieved during the synchronous input (compare Fig. $6 b 4, b 5$ with $a 4, a 5)$. These data indicate that a phasic inhibitory input alone, at least when mimicked as a $200 \mathrm{~Hz}$ sine wave, is not capable of providing the reproducibly invariant AP timing reported to be present during SPWs. Additionally, a relatively synchronous input pattern that was nevertheless subthreshold for dendritic spike initiation (50-90 inputs over 4 $\mathrm{ms}$ ) delivered to the dendrite in combination with the $200 \mathrm{~Hz}$ hyperpolarizing sine was also incapable of achieving the invariant AP output timing observed during dendritic spike initiation. AP timing during this input pattern varied across $100^{\circ}$ as the amount of input was varied from 50 to 90 inputs (Fig. $6 c 4, c 5$ ) and over $65^{\circ}$ with fluctuations in resting membrane potential $( \pm 5 \mathrm{mV})$ (data not shown).

Finally, the strict input pattern requirements of the nonlinear integration mode can be observed to provide a degree of functionality to a neuron that might be especially relevant to the precise sequential firing observed in the CA1 network during SPWs. When a neuron is presented with a spatially clustered and synchronous input pattern, together with a $200 \mathrm{~Hz}$ phasic proximal hyperpolarization, action potential output probability is high $(\sim 0.8)$ and a well timed output is observed (Fig. $7 b, d$, input labeled "clustered"). However, if a similarly sized input is distributed across spines covering $\sim 150 \mu \mathrm{m}$ of dendrite, the input efficacy is reduced (Fig. 3) and action potential output probability is likewise significantly inhibited $(\sim 0.15)$ (Fig. $7 d$ ). Furthermore, the precision of the spike timing for these few APs is quite poor and similar to that seen in Figure $6 c$. These data indicate that CA1 neurons can use the strict spatiotemporal requirements for dendritic spike generation to distinguish between multiple synchronous inputs, responding with a precise AP output only to input that is both synchronous and clustered. This would be useful if a particular CA1 neuron receives a succession of synchronous input from the different groups of CA3 pyramidal cells being sequentially read out during a single SPW/ripple event (Lee and Wilson, 2002). The ability to ignore altogether input that is not properly clustered (or at most produce an infrequent poorly timed output) will enhance the ability of the neuron b
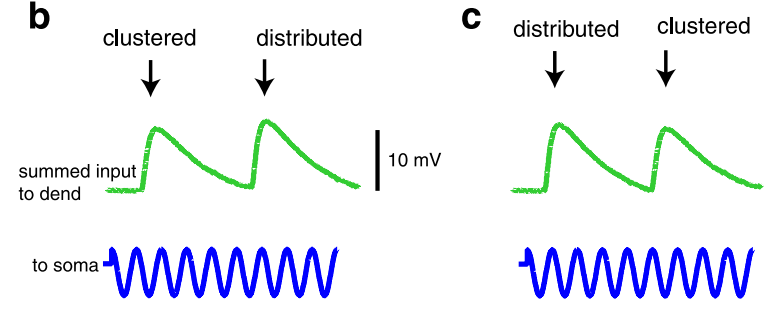

HWMW
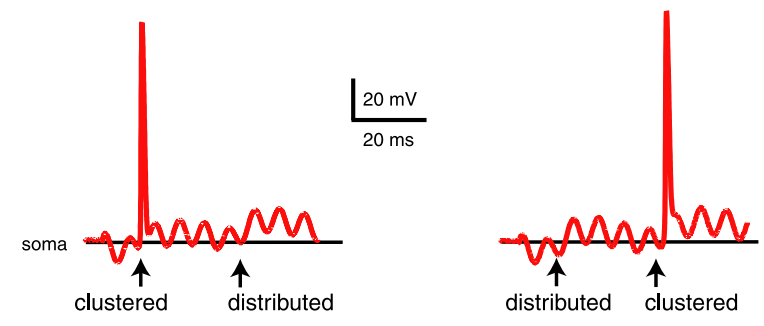

d

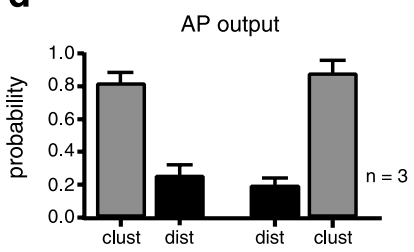

e

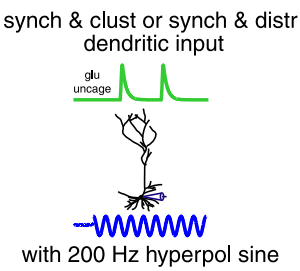

Figure 7. Nonlinear integration allows discrimination between synchronous inputs. $\boldsymbol{a}$, Two-photon image stack of a repre$\mu \mathrm{m})$ together with the somatic sine wave. When the clustered input pattern was delivered (arrow labeled "clustered"), a somatic and clustered input was much better at producing a well timed output than a synchronous but distributed input under recreated SPW conditions. Error bars indicate SE. e, Experimental setup.

to fire in the proper temporal order during the rapid network sequence replay.

Together, these data indicate that behaviorally relevant types of input patterns are capable of shifting the dendritic integration mode of CA1 pyramidal neurons and that this shift plays an important role in producing output patterns that resemble quite closely those associated with each of the two major behavioral states seen in the hippocampus. In light of these data, it seems likely that the characteristic network patterns associated with theta and SPW states are in part produced by this capability of CA1 pyramidal dendrites to change the way they integrate incoming input. Without this, it seems unlikely that the neurons embedded within the CA3-CA1 network could operate under such disparate timescales as those observed during theta and SPW states.

\section{Discussion}

In summary, we have directly demonstrated that different spatiotemporal patterns of synaptic input differentially engage the active properties of CA1 pyramidal dendrites to produce two distinct forms of integration. Furthermore, each of these dendritic integration modes induces a unique style of action potential output with significant functional relevance. Finally, we have attempted to associate this relationship between input patterns, 


\section{A) Theta state- input strength encoding}

extracellular theta wave $(5-10 \mathrm{~Hz})$
1) Asynchronous excit. synaptic input
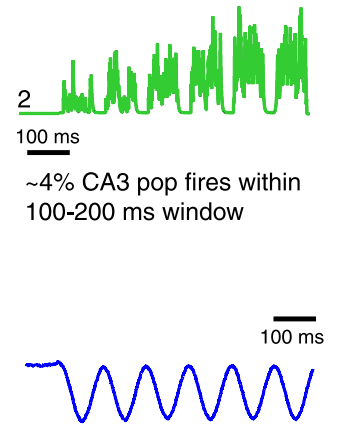

Phasic $(5-10 \mathrm{~Hz})$ inhibition to prox regions

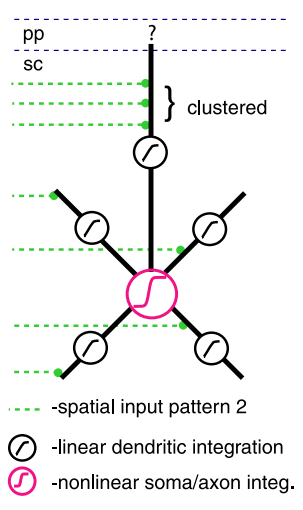

2) Dendrite performs linear integration

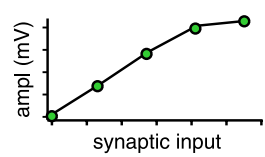

3) Output frequency and timing vary as function of input strength

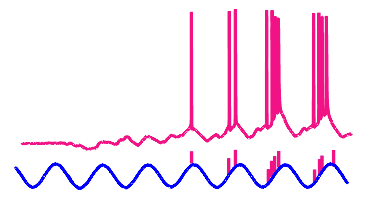

\section{B) Sharp wave state- feature detection}

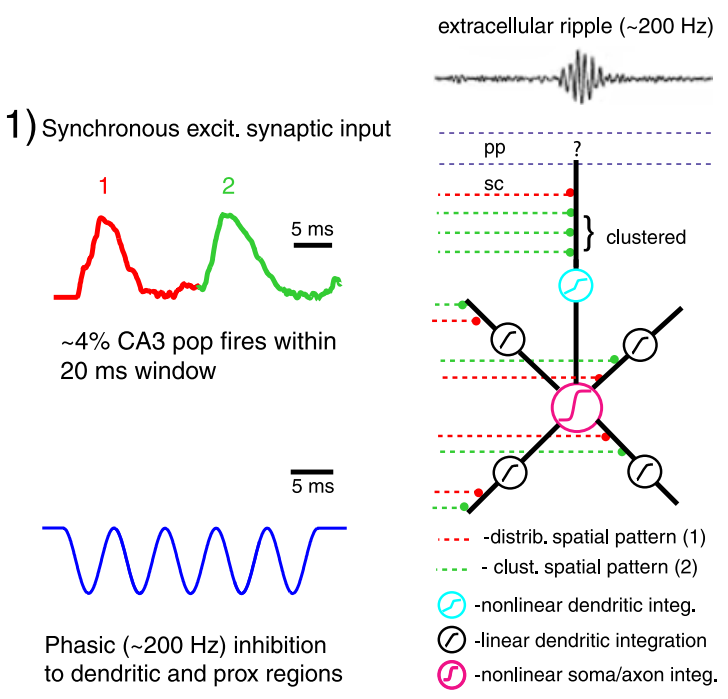

Dendritic compartment receiving

2) clustered input (2) performs supra-linear integration

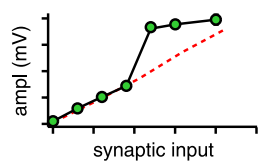

3) Precise output is invariant during a single 1-2 ms phase of ripple

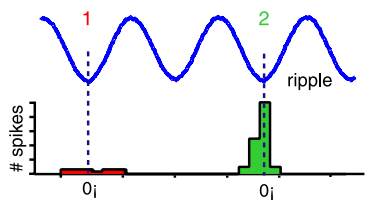

Figure 8. Model of state-dependent dendritic computations. $\boldsymbol{A}$, During theta states (characteristic extracellular population pattern shown), excitatory input (A1) to the dendritic arbor is relatively asynchronous, increasing steadily as the animal moves toward and through the place field of the cell. Inhibitory input to the proximal regions of the neuron (proximal trunk, soma, and axon initial segment) is phasic at $5-10 \mathrm{~Hz}$ (blue trace). Inhibition to stratum radiatum dendritic regions is similarly phasic but shifted $\sim 180^{\circ}$, increasing the phasic profile of dendritic depolarization. $\boldsymbol{A 2}, \boldsymbol{A 3}$, During such input conditions, the dendritic arbor will remain in a linear integration mode $(\boldsymbol{A 2})$, and action potential output rate during a single theta cycle will increase and timing will shift forward (phase precession) as the number of inputs increases with the position of the animal (A3). Red ticks on the sine wave below the output trace show the AP number and timing relative to the theta phase. The cartoon neuron shows that, in this behavioral state, dendritic integration is linear (black linear function), allowing it to transfer a level of depolarization that is a linear function of the number of inputs to the soma/axonal output AP generating zone (purple sigmoid function) even as a significant subset of the input is spatially clustered onto one dendritic compartment. $\boldsymbol{B}$, During sharp-wave states (characteristic extracellular population pattern shown), excitatory input (B1) to the dendritic arbor is $\sim 10$-fold more synchronous and the phasic inhibitory input to the proximal regions is at a much higher rate $(200 \mathrm{~Hz}$; blue trace). Dendritic inhibition has similar timing as that of proximal inhibition and should again enhance the phasic nature of the dendritic depolarization. CA1 neurons may receive multiple synchronous inputs (patterns 1 and 2; red and green, respectively) during a single ripple event. B2, B3, During such input conditions, only the dendritic compartment receiving clustered input will shift into a supralinear integration mode (B2), and action potential output will repeatedly occur precisely during the brief time period of least proximal inhibition for only the clustered pattern (B3). The cartoon neuron shows the dendritic compartment receiving clustered input is integrating nonlinearly (light blue sigmoid function), allowing it to transfer a relatively constant amount of rapid depolarization to the soma/axonal output AP generating zone (purple sigmoid function). This increases the temporal output precision while reducing its sensitivity to input strength. Green and red dashed lines represent Schaffer collateral input that is either clustered or distributed, respectively. The blue dashed lines are perforant path. dendritic integration mode, and output generation with the two main behavioral states found in the hippocampus. In this respect, we have shown that asynchronous input patterns similar to those found during theta states summate linearly in the dendrites and soma, ultimately producing an action potential output whose rate and coarse timing is a direct function of the amount of input. This is an input-output relationship that closely approximates what is observed in the rodent hippocampus during active exploration of space. In contrast, synchronous inputs that are also clustered in space summate supralinearly through the initiation of a dendritic spike that then propagates actively to the soma/ axon. This actively propagating dendritic spike evokes a precise axonal output that reflects the spatiotemporal pattern of the input. This integration mode appears to be uniquely capable of producing the precise level of spike timing required by the muchcompressed timescales present during sharp wave states. There is in fact some evidence of dendritic spiking during SPWs (Kamondi et al., 1998). Together, these data indicate that CA1 pyramidal neurons can perform two very different single-cell computations, depending on the pattern of activity within the network of presynaptic CA3 cells (Fig. 8).

\section{Dendritic properties involved in linear and nonlinear integration modes} The dendritic and synaptic properties of CA1 pyramidal neurons allow them to integrate synaptic inputs in either an essentially linear or highly nonlinear manner depending on the type of stimuli received. The linear integration mode is remarkable in that it is an "active linearization" achieved as $\mathrm{Na}^{+}$, NMDA, $I_{\mathrm{h}}$, and $\mathrm{K}^{+}$ channel activations effectively counterbalance each other and the loss of AMPA current driving force over a window of dendritic $V_{\mathrm{m}}$ spanning approximately from -70 to $-25 \mathrm{mV}$. Indeed, blockade of any one of these channel populations dramatically alters the dendritic current-voltage relationship (Cash and Yuste, 1999; Gasparini et al., 2004). In this study, we have used the distal apical trunk as a model dendritic compartment, because its morphology allows for direct electrical recordings and because of the availability of highquality published data. Preliminary data from our lab support the idea that other dendritic regions, such as the radial oblique and basal branches, possess very similar functional properties (Magee and Losonczy, 2004).

The ability of a particular input pattern 
to produce the transition from linear to supralinear integration depends on the rate of membrane depolarization produced by the input. Indeed, local dendritic spike threshold becomes more depolarized as $d V / d t$ decreases, eventually reaching such depolarized levels that no dendritic spikes can be initiated (Gasparini et al., 2004). This property, combined with the small amplitude and short duration of unitary EPSPs in the dendrite $(<1 \mathrm{mV} ;<10 \mathrm{~ms}$ half-width) (Magee and Cook, 2000; Smith et al., 2003) determine that only highly synchronized and spatially clustered inputs will provide the level of rapid dendritic depolarization required (Turner et al., 1991; Golding and Spruston, 1998). In contrast, as long as the pattern of input is relatively distributed in space or time, the lower and more stable AP threshold at the output region will be exceeded. Thus, very specific input conditions are required to produce the shift from linear to nonlinear integration, and unless these are met, the arbor will usually operate in a linear integration mode.

An additional factor to be considered is the neuromodulatory state of the neuron. It is currently thought that theta states are associated with relatively high levels of $\mathrm{ACh}$, whereas norepinephrine levels have been reported to be high during SPW activity (for review, see Pace-Schott and Hobson, 2002). Because dendritic ion channels are susceptible to these neuromodulators (Tsubokawa and Ross, 1997; Colbert and Johnston, 1998; Hoffman and Johnston, 1998; Gasparini and Magee, 2002; Magee and Johnston, 2005), the exact spatiotemporal patterns of the input required to shift the processing mode may vary from one behavioral state to another. Moreover, activity-dependent processes that modify dendritic active channels (Frick et al., 2004) could produce similar alterations in the pattern dependence of dendritic integration.

Finally, dendritic spike propagation has been reported to be variable, with some spikes propagating more strongly than others (Golding and Spruston, 1998; Ariav et al., 2003; Gasparini et al., 2004). Obviously, weakly propagating local spikes will not be as effective as stronger spikes in shifting the output pattern of the neuron. However, because the forward propagation of dendritic spikes is very sensitive to subthreshold levels of depolarization (Ariav et al., 2003; Gasparini et al., 2004; Jarsky et al., 2005), background excitatory input may provide a mechanism to equalize the impact of most dendritic spikes.

\section{Dendritic computations during different behavioral states}

The distinct input-output patterns found during theta and SPW states are indicative of the very different functions thought to occur during the respective behavioral states. It is currently believed that the temporal sequences of a particular episode occurring over seconds of time are encoded and transiently stored within the hippocampal network during theta states (McNaughton and Morris, 1987; Mehta et al., 2000; Dragoi et al., 2003). During subsequent SPW states, the previously stored information seems to be "read out" to the higher cortical areas for consolidation as a tightly compressed replay (now over only tens of milliseconds) of essentially the same neuronal firing sequences activated during previous theta states (Qin et al., 1997; Siapas and Wilson, 1998; Kudrimoti et al., 1999; Nadasdy et al., 1999; Lee and Wilson, 2002). It is our hypothesis that, as the hippocampus moves from one state to another, a switch in the mode of dendritic integration occurs that allows CA1 pyramidal neurons (and perhaps other hippocampal neurons) to perform the computation that is most appropriate for the unique timescales present during each behavioral state.

Our data suggest that, during theta states, the regions of the neuron receiving Schaffer collateral input should operate in a linear integration mode with all input contributing to the output of the cell (Magee, 2000). Linear integration models have been shown to be quite effective in producing output patterns (changes in firing frequency) that accurately reflect changes in the quantity of inputs (termed "power variation") (Poirazi et al., 2003). The addition of proximal phasic inhibitory input permits CA1 pyramidal neurons to also use spike timing to encode the location of the animal in space (Harris et al., 2002; Mehta et al., 2002; Huxter et al., 2003). Thus, during theta states, the pyramidal cell network may be in an integration mode that allows the cells to calculate power variations that encode the sequence of an animal's behavior within the rate and course timing of neuronal firing over timescales that are dictated by the actual behavior (many hundreds of milliseconds). This type of cellular activity may be most advantageous for producing the synaptic and dendritic plasticity required for transient information storage within the hippocampal network. The functioning of these neurons during this time should not be confused with the concept of a "point neuron" simply because the dendrites are linearly integrating synaptic input. Indeed the presence of regionally localized inhibition and excitation as well as the complex interplay between backpropagating spikes and synaptic depolarization are obvious interactions among proximal and distal compartments that would not be present within a point neuron.

Processing in the nonlinear mode could functionally separate the CA1 arbor into a large number of independent nonlinear computational units, each sending its own output to the soma (Poirazi and Mel, 2001; Wei et al., 2001). This form of integration would allow neurons to respond not so much to the quantity of input but instead to the specific spatiotemporal input pattern (termed "configuration variability") (Poirazi et al., 2003). During the period of heightened synchrony found during SPW states, the lack of an actual constraining behavior allows the network to process essentially the same information concerning the sequence of events $\sim 20$ times more rapidly (tens of milliseconds) (Nadasdy et al., 1999; Lee and Wilson, 2002). The challenge to the individual CA1 neuron within the network is to reproducibly fire APs during a brief time window within the correct ripple phase so that the appropriate firing order among the neurons encoding the sequence can be maintained. Furthermore, this precise firing pattern has to occur many times over as the same neuronal sequences are repeatedly replayed (Lee and Wilson, 2002). The features provided by the nonlinear integration mode allow the postsynaptic CA1 pyramidal neuron to properly respond to the demands imposed on it during SPW states. That is, the cell is not likely to fire until it receives the appropriately timed and clustered input pattern (the proper configuration), at which point the neuron will respond with an extraordinarily well timed and invariant output. The rapid replay and exquisite network timing found during SPWs may facilitate the induction of plasticity in downstream cortical structures, perhaps through the induction of dendritic spiking and nonlinear dendritic integration in those cells.

In conclusion, there appears to be a direct link between the specific synaptic input pattern, the mode of dendritic integration, and ultimately the form of action potential output evoked in hippocampal CA1 pyramidal neurons. Furthermore, the features of the output deriving from the different integration modes appear to be reminiscent of those found during the two main hippocampal behavioral states. This link between input, integration, and output may allow the most appropriate single-cell computation to be performed during each behavioral state. 


\section{References}

Allen C, Stevens CF (1994) An evaluation of causes for unreliability of synaptic transmission. Proc Natl Acad Sci USA 91:10380-10383.

Ariav G, Polsky A, Schiller J (2003) Submillisecond precision of the inputoutput transformation function mediated by fast sodium dendritic spikes in basal dendrites of CA1 pyramidal neurons. J Neurosci 23:7750-7758.

Bannister NJ, Larkman AU (1995) Dendritic morphology of CA1 pyramidal neurones from the rat hippocampus. II. Spine distributions. J Comp Neurol 360:161-171.

Buzsaki G (1986) Hippocampal sharp waves: their origin and significance. Brain Res 398:242-252.

Canals S, Lopez-Aguado L, Herreras O (2005) Synaptically recruited apical currents are required to initiate axonal and apical spikes in hippocampal pyramidal cells: modulation by inhibition. J Neurophysiol 93:909-918.

Cash S, Yuste R (1999) Linear summation of excitatory inputs by CA1 pyramidal neurons. Neuron 22:383-394.

Cobb SR, Buhl EH, Halasy K, Paulsen O, Somogyi P (1995) Synchronization of neuronal activity in hippocampus by individual GABAergic interneurons. Nature 378:75-78.

Colbert CM, Johnston D (1998) Protein kinase C activation decreases activity-dependent attenuation of dendritic $\mathrm{Na}^{+}$current in hippocampal CA1 pyramidal neurons. J Neurophysiol 79:491-495.

Csicsvari J, Hirase H, Czurko A, Buzsaki G (1998) Reliability and state dependence of pyramidal cell-interneuron synapses in the hippocampus: an ensemble approach in the behaving rat. Neuron 21:179-189.

Csicsvari J, Hirase H, Czurko A, Mamiya A, Buzsaki G (1999) Oscillatory coupling of hippocampal pyramidal cells and interneurons in the behaving rat. J Neurosci 19:274-287.

Csicsvari J, Hirase H, Mamiya A, Buzsaki G (2000) Ensemble patterns of hippocampal CA3-CA1 neurons during sharp wave-associated population events. Neuron 28:585-594.

Dragoi G, Harris KD, Buzsaki G (2003) Place representation within hippocampal networks is modified by long-term potentiation. Neuron 39:843-853.

Fox SE (1989) Membrane potential and impedance changes in hippocampal pyramidal cells during theta rhythm. Exp Brain Res 77:283-294.

Frick A, Magee J, Johnston D (2004) LTP is accompanied by an enhanced local excitability of pyramidal neuron dendrites. Nat Neurosci 7:126-135.

Gasparini S, Magee JC (2002) Phosphorylation-dependent differences in the activation properties of distal and proximal dendritic $\mathrm{Na}^{+}$channels in rat CA1 hippocampal neurons. J Physiol (Lond) 541:665-672.

Gasparini S, Migliore M, Magee JC (2004) On the initiation and propagation of dendritic spikes in CA1 pyramidal neurons. J Neurosci 24:11046-11056.

Golding NL, Spruston N (1998) Dendritic sodium spikes are variable triggers of axonal action potentials in hippocampal CA1 pyramidal neurons. Neuron 21:1189-1200.

Golding NL, Jung HY, Mickus T, Spruston N (1999) Dendritic calcium spike initiation and repolarization are controlled by distinct potassium channel subtypes in CA1 pyramidal neurons. J Neurosci 19:8789-8798.

Harris KD, Henze DA, Hirase H, Leinekugel X, Dragoi G, Czurko A, Buzsaki G (2002) Spike train dynamics predicts theta-related phase precession in hippocampal pyramidal cells. Nature 417:738-741.

Harsch A, Robinson HP (2000) Postsynaptic variability of firing in rat cortical neurons: the roles of input synchronization and synaptic NMDA receptor conductance. J Neurosci 20:6181-6192.

Hoffman DA, Johnston D (1998) Downregulation of transient $\mathrm{K}^{+}$channels in dendrites of hippocampal CA1 pyramidal neurons by activation of PKA and PKC. J Neurosci 18:3521-3528.

Hoffman DA, Magee JC, Colbert CM, Johnston D (1997) $\mathrm{K}^{+}$channel regulation of signal propagation in dendrites of hippocampal pyramidal neurons. Nature 387:869-875.

Huxter J, Burgess N, O'Keefe J (2003) Independent rate and temporal coding in hippocampal pyramidal cells. Nature 425:828-832.

Jarsky T, Roxin A, Kath W, Spruston N (2005) Conditional dendritic spike propagation following distal synaptic activation of hippocampal CA1 pyramidal neurons. Nat Neurosci 8:1667-1676.

Kamondi A, Acsady L, Buzsaki G (1998) Dendritic spikes are enhanced by cooperative network activity in the intact hippocampus. J Neurosci 18:3919-3928.

Klausberger T, Magill PJ, Marton LF, Roberts JD, Cobden PM, Buzsaki G,
Somogyi P (2003) Brain-state- and cell-type-specific firing of hippocampal interneurons in vivo. Nature 421:844-848.

Klausberger T, Marton LF, Baude A, Roberts JD, Magill PJ, Somogyi P (2004) Spike timing of dendrite-targeting bistratified cells during hippocampal network oscillations in vivo. Nat Neurosci 7:41-47.

Kudrimoti HS, Barnes CA, McNaughton BL (1999) Reactivation of hippocampal cell assemblies: effects of behavioral state, experience, and EEG dynamics. J Neurosci 19:4090-4101.

Larkum ME, Zhu JJ, Sakmann B (2001) Dendritic mechanisms underlying the coupling of the dendritic with the axonal action potential initiation zone of adult rat layer 5 pyramidal neurons. J Physiol (Lond) 533:447-466.

Lee AK, Wilson MA (2002) Memory of sequential experience in the hippocampus during slow wave sleep. Neuron 36:1183-1194.

Magee JC (1998) Dendritic hyperpolarization-activated currents modify the integrative properties of hippocampal CAl pyramidal neurons. J Neurosci 18:7613-7624.

Magee JC (2000) Dendritic integration of excitatory synaptic input. Nat Rev Neurosci 1:181-190.

Magee JC, Carruth M (1999) Dendritic voltage-gated ion channels regulate the action potential firing mode of hippocampal CA1 pyramidal neurons. J Neurophysiol 82:1895-1901.

Magee JC, Cook EP (2000) Somatic EPSP amplitude is independent of synapse location in hippocampal pyramidal neurons. Nat Neurosci 3:895-903.

Magee JC, Johnston D (1997) A synaptically controlled, associative signal for Hebbian plasticity in hippocampal neurons. Science 275:209-213.

Magee JC, Johnston D (2005) Plasticity of dendritic function. Curr Opin Neurobiol 15:334-342.

Magee JC, Losonczy A (2004) An investigation of the input-output properties of radial oblique dendrites in hippocampal CA1 pyramidal neurons. Soc Neurosci Abstr 30:737.12.

Margulis M, Tang C (1998) Temporal integration can readily switch between sublinear and supralinear summation. J Neurophysiol 79:2809-2813.

McDermott C, Hardy M, Bazan N, Magee JC (2005) Mechanisms of altered synaptic plasticity induced by sleep deprivation in hippocampal CA1 neurons. J Physiol (Lond), in press.

McNaughton BL, Morris RGM (1987) Hippocampal synaptic enhancement and information storage within a distributed memory system. Trends Neurosci 10:408-415.

Megias M, Emri Z, Freund TF, Gulyas AI (2001) Total number and distribution of inhibitory and excitatory synapses on hippocampal CA1 pyramidal cells. Neuroscience 102:527-540.

Mehta MR, Quirk MC, Wilson MA (2000) Experience-dependent asymmetric shape of hippocampal receptive fields. Neuron 25:707-715.

Mehta MR, Lee AK, Wilson MA (2002) Role of experience and oscillations in transforming a rate code into a temporal code. Nature 417:741-746.

Metz AE, Jarsky T, Martina M, Spruston N (2005) R-type calcium channels contribute to afterdepolarization and bursting in hippocampal CA1 pyramidal neurons. J Neurosci 25:5763-5773.

Nadasdy Z, Hirase H, Czurko A, Csicsvari J, Buzsaki G (1999) Replay and time compression of recurring spike sequences in the hippocampus. J Neurosci 19:9497-9507.

Nettleton JS, Spain W (2000) Linear to supra-linear summation of AMPAmediated EPSPs in neocortical pyramidal neurons. J Neurophysiol 83:3310-3322.

O'Keefe J, Dostrovsky J (1971) The hippocampus as a spatial map. Preliminary evidence from unit activity in the freely-moving rat. Brain Res 34:171-175.

O'Keefe J, Nadel L (1978) The hippocampus as a cognitive map. Oxford: Clarendon.

O’Keefe J, Recce ML (1993) Phase relationship between hippocampal place units and the EEG theta rhythm. Hippocampus 3:317-330.

Pace-Schott EF, Hobson JA (2002) The neurobiology of sleep: genetics, cellular physiology and subcortical networks. Nat Rev Neurosci 3:591-605.

Poirazi P, Mel BW (2001) Impact of active dendrites and structural plasticity on the memory capacity of neural tissue. Neuron 29:779-796.

Poirazi P, Brannon T, Mel BW (2003) Pyramidal neuron as two-layer neural network. Neuron 37:989-999.

Polsky A, Mel BW, Schiller J (2004) Computational subunits in thin dendrites of pyramidal cells. Nat Neurosci 7:621-627. 
Qin YL, McNaughton BL, Skaggs WE, Barnes CA (1997) Memory reprocessing in corticocortical and hippocampocortical neuronal ensembles. Philos Trans R Soc Lond B Biol Sci 352:1525-1533.

Raastad M, Storm JF, Andersen P (1992) Putative single quantum and single fibre excitatory postsynaptic currents show similar amplitude range and variability in rat hippocampal slices. Eur J Neurosci 4:113-117.

Siapas AG, Wilson MA (1998) Coordinated interactions between hippocampal ripples and cortical spindles during slow-wave sleep. Neuron 21:1123-1128.

Singer W (1999) Neuronal synchrony: a versatile code for the definition of relations? Neuron 24:49-65.

Smith MA, Ellis-Davies GC, Magee JC (2003) Mechanism of the distancedependent scaling of Schaffer collateral synapses in rat CA1 pyramidal neurons. J Physiol (Lond) 548:245-258.

Spruston N, Kath WL (2004) Dendritic arithmetic. Nat Neurosci 7:567-569.

Tamas G, Szabadics J, Somogyi P (2002) Cell type- and subcellular positiondependent summation of unitary postsynaptic potentials in neocortical neurons. J Neurosci 22:740-747.
Tsubokawa H, Ross WN (1997) Muscarinic modulation of spike backpropagation in the apical dendrites of hippocampal CA1 pyramidal neurons. J Neurosci 17:5782-5791.

Turner RW, Meyers DE, Richardson TL, Barker JL (1991) The site for initiation of action potential discharge over the somatodendritic axis of rat hippocampal CA1 pyramidal neurons. J Neurosci 11:2270-2280.

Vanderwolf CH (1969) Hippocampal electrical activity and voluntary movement in the rat. Electroencephalogr Clin Neurophysiol 26:407-418.

Wei DS, Mei YA, Bagal A, Kao JP, Thompson SM, Tang CM (2001) Compartmentalized and binary behavior of terminal dendrites in hippocampal pyramidal neurons. Science 293:2272-2275.

Wilson MA, McNaughton BL (1994) Reactivation of hippocampal ensemble memories during sleep. Science 265:676-679.

Ylinen A, Bragin A, Nadasdy Z, Jando G, Szabo I, Sik A, Buzsaki G (1995) Sharp wave-associated high-frequency oscillation $(200 \mathrm{~Hz})$ in the intact hippocampus: network and intracellular mechanisms. J Neurosci $15: 30-46$. 